\title{
O supervisor e as estratégias educacionais dos encontros locorregionais no Programa Mais Médicos do Brasil: reflexões acerca de concepções e práticas.
}

\section{The supervisor andeducationalstrategiesof meetings regional-local in the More DoctorsProgramofBrazil: reflectionsconcerningconceptsandpractices.}

\section{El supervisor y estrategias educativas de reunioneslocorregionalenel Programa Más Médicos de Brasil: reflexiones sobre conceptos y prácticas.}

\author{
Elyne Montenegro Engstrom ${ }^{1}$ \\ Luis Claudio de Carvalho \\ Valéria Ferreira Romano \\ Andrea A C Castro
}

RESUMO: Para aprimorar a formação médica, o Programa Mais Médicos do Brasil (PMM), dentre uma de suas finalidades, realizou a provisão de médicos nas regiões prioritárias para o SUS, a fim de reduzir as desigualdades regionais em saúde, integrando ensino-serviço, com a supervisão direta aos médicos participantes. O presente artigo discute uma experiência de supervisão coletiva (encontros locorregionais) desenvolvida em um município do estado do Rio de Janeiro em 2014-2015. Ao se considerar a potência de desenvolver a força de trabalho do SUS segundo pressupostos da educação permanente em saúde, procurou-se refletir acerca da identificação de necessidades educacionais, da construção de estratégias e ofertas de formação no ambiente do trabalho dos médicos do PMM. Foram realizados sete encontros, com a participação de 16 médicos participantes do PMM, além de supervisores (2), tutor, gestor municipal e apoiadores/especialistas municipais. Metodologias baseadas na pedagogia da problematização foram vivenciadas e as temáticas foram o diagnóstico situacional e identificação de prioridades (matriz de problemas/propostas), sendo eleitas as áreas: saúde mental, uso de drogas, saúde da mulher/pré-natal e criança. As estratégias educacionais facilitaram a integração da gestão, supervisãoe médicos, em diálogo com às necessidades de saúde

1 Médica, doutorado em saúde pública, pesquisadora e coordenadora do mestrado profissional em atenção primária/ ENSP/Fiocruz.E-mail: elyneengstrom@gmail.com 
da população, o modelo de atenção e a organização da rede de saúde local.

Palavras chave: educação permanente,educação em saúde, prática profissional, atenção primária à saúde,aprendizagem baseada em problema.

ABSTRACT: To improve the medical training, the "More Doctors Program of Brazil" (MDP), including one of his purposes, performed the medical supply priority regions for the Universal Health System (UHS), in order to reduce regional inequalities in health, integrating teaching and service, with direct supervision to participants. This article discusses a collective supervisory experience (meetings regional-local) developed in a municipality in the State of Rio de Janeiro in 2014-2015. When you consider the power to develop the workforce of the UHS according to assumptions of permanent education in health, sought to reflect about the identification of educational needs, building strategies and training offerings in the work environment of the doctors of the MDP. Seven meetings were held with the participation of 16 doctors participating in the PMM, along with supervisors (2), tutor, City Manager and supporters/municipal experts. Methodologies based on pedagogy of questioning were experienced and the themes were the situational diagnosis and identification of priorities (array of issues/proposals), being elected the areas: mental health, drug use, women's health/prenatal care and child. The construction of educational strategies were based on the integration of management, supervision and doctors, in dialogue with the health needs of the population, the model of attention and organization of local health network.

Key words: permanenteducation, healtheducation, professional practice,primaryhealthcare,probl em-basedlearning.

RESUMEN: Para mejorar la formación médica, el "Programa Más Médicos de Brasil”(PMM), incluyendo uno de sus propósitos, realizaronlasregionesprioritarias de suministros médicos para SUS, para reducirlas desigualdades regional es en salud, integración de laenseñanza y servicio, condirigensupervisión de los participantes. Este artículo aborda una experiência de supervisióncolectiva (reunioneslocorregionais) se convirtióenunmunicipioenel estado de Río de Janeiroen 2014-2015. Si tenemosencuentael poder de desarrollarlafuerza de trabajo de SUS segúnhipótesis de laeducación permanente ensalud, intentaron reflexionar acerca de laidentificación de necesidades educativas, construcción de estrategias y ofertas de capacitacióneneltrabajo de losdoctores de lo PMM. Se celebraronsietereuniones, conlaparticipación de 16 doctoresenel PMM, junto conlasupervisión (2), tutor, administrador de laciudad y los expertos y gestión municipal. Metodologíasbasadasenlapedagogía de laproblematizaciónfueron experimentados y los temas fueronel diagnóstico situacional y laidentificación de prioridades (amplia gama de temas o propuestas), siendo elegido las áreas de: salud mental, consumo de drogas, salud/controlprenatal de lamujer y niño. La construcción de estrategias educativas se basaenlaintegración de lagestión, supervisión y educación, en diálogo conlasnecesidades de salud de lapoblación, el modelo de atención y organización de red de salud local.

Palabras clave: educaciónpermanente,educación para lasalud,prácticaprofessional,atención primaria de salud,aprendizajebasadoen problemas 


\section{INTRODUÇÃO}

Nos países que adotam sistemas de saúde de caráter universal, a atenção primária à saúde (APS) é base constitutiva, estando assim, na centralidade das agendas políticas de saúde. No contexto brasileiro, a APS configura-se como um dos centros do debate sobre os desafios para o fortalecimento do SUS, ao buscar a implementação de seus atributos essenciais e ao apresentar-se como reorientadora do modelo de atenção ${ }^{1}$. Neste cenário, pode-se afirmar que um destes desafios, é a provisão de força de trabalho qualificada para lidar com as necessidades de saúde das populações.

Tendo como uma de suas finalidades formar recursos humanos na área médica para o SUS, o Programa Mais Médicos do Brasil (PMM) foi instituído pela Medida Provisória $N^{0} 621$ de 8 de julho de 2013 sob gestão do Ministério da Educação e da Saúde. ${ }^{2}$ Assumiu como objetivos, fortalecer a prestação de serviços na atenção básica, com investimentos em infraestrutura das unidades básicas de saúde (UBS), aprimorar a formação médica no país e na vertente denominada Projeto Mais Médicos, visava ainda a provisão de médicos nas regiões prioritárias, a fim de reduzir as desigualdades regionais em saúde.

Até 2015, nos cinco ciclos de provisão do Projeto, 18.240 médicos vem atuando em 34 Distritos Sanitários Especiais Indígenas e em 4.058 municípios brasileiros, cobrindo 72,8\% das cidades, e atendendo 63 milhões de pessoas que antes encontravam algum grau de obstáculo no acesso ao SUS, especialmente na Atenção Básica. ${ }^{3,4}$ As modalidades de participantes incluem: médicos formados no Brasil ou com diploma revalidado no país; médicos brasileiros formados em medicina no exterior, com habilitação para o exercício da medicina no exterior e médicos estrangeiros com habilitação para o exercício da medicina fora do país (intercambistas). ${ }^{2}$ Para todas as modalidades, a participação no PMM ficou condicionada ao aperfeiçoamento dos médicos por meio de realização de um curso de especialização ofertado por instituição pública de educação superior onde o ensino, a pesquisa e a extensão, integrariam o ensino com o serviço.

Pressupõe-se que este processo de educação em saúde estejafundamentado pelas diretrizes pedagógicas da Educação Permanente ${ }^{5}$ e contribua para que os médicos adquiriam as competências esperadas para profissionais de nível superior que atuam na Estratégia de Saúde da Família, normatizações pelo Ministério da Saúde. ${ }^{6}$ Para além do curso de especialização, o PMM previa a existência de outras atividades formativas, como a supervisão aos médicos participantesem seu ambiente de trabalho.Na estrutura de integrantes do Projeto existe, além do médico participante, um supervisor e um tutor. O supervisor é um profissional médico responsável pela supervisão contínua e permanente do médico participante e o tutor um docente médico responsável pela orientação acadêmica e pelo planejamento das atividades do supervisor, garantindo assim supervisão médica e tutoria acadêmica a todos os médicos do Projeto. ${ }^{2}$ Cada tutor é responsável por 10 supervisores e cada supervisor por um máximo de 10 médicos. 
Dessa forma, em cada estado, uma instituição federal de ensino superior foi credenciada como responsável pelo apoio à formação em serviço, sendo a Universidade Federal do Rio de Janeiro (UFRJ), instituição responsável pela tutoria e supervisão no referido Estado desde setembro de 2013.

No que se refere às atribuições do supervisor, estabeleceu-se como metodologia de trabalho, a realização de visitas ao médico participante, ao menos uma vez ao mês,nas unidades básicas de saúde (UBS). Estas objetivavam a supervisão clínica individual, a avaliação das condições de estrutura das UBS, os aspectos gerenciais/administrativos do processo de trabalho, da relação com a equipe, a comunidade e a gestão municipal.

Recomendou-se, ainda, a realização de encontros ou reuniões a cada dois/três meses com o coletivo de participantes do programa - denominados encontros locorregionais - que poderiam agregar médicos de um ou mais municípios próximos, de acordo com o número de profissionais ou aspectos logísticos (horários, localização, afinidade de temas e prioridades, etc.). Estes momentos teriam a potencialidade de promover trocas de experiências, assim como compartilhar conhecimentos científicos e técnicos pertinentes ao trabalho na atenção primária à saúde.

Sabe-se que a APS desempenha papel diferenciado dos demais níveis de atenção à saúde em função das características clínicas dos pacientes e de seus problemas, assim como dos contextos sociais dos territórios sob sua responsabilidade ${ }^{7}$, que se expressam na pluralidade dos cenários de práticas. Trazer tal diversidade e a experiência vivenciada, o saber e a inteligênciaproduzida pelos profissionais no cotidiano de seu trabalho na APS é fundamental para os processos formativos, como nos lembraCeccin (2012). ${ }^{8}$ Ainda segundo o autor, para o desenvolvimento de competências para o trabalho em saúde, as políticas públicas de educação na saúde reconhecem a insuficiência dos treinamentos formais e teóricos, propondo-se cada vez mais estratégias de compartilhamento de saberes e experiências ou as rodas de conversa como instrumentos pedagógicos.

Nos materiais técnicos e orientações acerca do PMM, percebe-se uma maior ênfase aos aspectos operacionais do papel do supervisor e carência de reflexões acerca de metodologias e/ou estratégias de trabalho, em especial no campo da educação em saúde. Há, por exemplo, normativas de periodicidade de contato supervisor-médico participante (ao menos uma vez ao mês), de forma presencial (excepcionalmente a distância), de preenchimento mensal de um roteiro de entrevista (relatório de supervisão de primeira visita e/ou de visitas de acompanhamento), atividades acompanhadasem plataforma eletrônica da Universidade Aberta do SUS (denominadoweb portfolio).

Considerando a escassez de publicações acerca da atuação do supervisor do PMM, especialmente de suas estratégias educacionais para lidar com a diversidade de práticas na APS, o presente artigo discute uma experiência de supervisão coletiva (locorregional) desenvolvida emum município do 
estado do Rio de Janeiro. Ao se considerar a potência de desenvolver a força de trabalho do SUS para qualificar a atenção à saúde, segundo pressupostos da educação permanente em saúde, procurou-se refletir acerca da identificação de necessidades educacionais, da construção de estratégias e ofertas de formação no ambiente do trabalho dos referidos médicos.

\section{Descrevendo nosso cenário}

O PMM no estado do Rio de Janeiro, em maio de 2015, contava com 736 médicos, atuando em 74 dos 92 municípios do estado, perfazendo 80,4\% de cobertura, proporção elevada se comparada à do país. ${ }^{9} \mathrm{O}$ estudo deste cenário é, pois, relevante se considerado ainda, os inúmeros desafios dos centros urbanos para expansão e consolidação do modelo da APS integral e robusta.A experiência aqui apresentada foi conduzida no município de Niterói, situado na região metropolitana do RJ, no período de março de 2013 a setembro de 2015, contando com a participação direta dos autores do presente artigo na função de supervisores ou tutores no período. Segundo o IBGE (2014) ${ }^{10}$, Niterói tem população estimada de 495.470 habitantes, com o Índice de Desenvolvimento Humano de 0,837 , considerado muito alto, embora apresenta bolsões de pobreza e de grande risco social, reflexo da desigualdade social.A violência urbana, tráfico de drogas e uso de armas de fogo tem sido problemas relevantes, especialmente nas comunidades mais desfavorecidas socialmente.

Inspirado no modelo cubano de atenção básica, o município é pioneiro no Programa de Médico da Família (PMF) no Brasil, iniciado no ano de 1992 e recebeu seu primeiro médico do PMM em outubro de 2013, um intercambista (médico estrangeiro ou brasileiro formado no exterior). Segundo informações da gestão local do PMM, até setembro de 2015, 2 supervisores e 16 novos médicos atuavam no PMM, sendo dois brasileiros formados no Brasil; três brasileiros formados no exterior; nove médicos cubanos; e dois médicos estrangeiros formados no exterior, contingente que representa $25 \%$ dos médicos da atenção básica do município. Estes profissionais estão alocados em lugares de difícil acesso e/ou áreas de grande risco social.

\section{Encontros locorregionais: concepçõespedagógicas}

Se uma pedagogia de transmissão tem sido hegemonicamente utilizada como opção pedagógica na formação educacional do país e na maioria dos países ocidentais, não seria difícil pensar que isto se reproduz nas Escolas Médicas. As maiores consequências individuais e sociais previsíveis de uma pedagogia de transmissão seriam, segundo Bordenave $(1977)^{11}$ : elevada absorção de informação, memorização excessiva, aluno passivo com pouca atitude crítica, hipervalorizarão do professor ou de textos como fonte de informação, distanciamento entre teoria e prática, tendência ao racionalismo radical, preferência por especulação teórica e pouca problematização da realidade.

Na contramão desta intencionalidade, adotamos a Pedagogia da Problematização, fundamentada na literatura nacional e internacional, ${ }^{12,13,14,15}$ como proposta pedagógica no PMMno município. A Pedagogia da Problematização estimula o surgimento de um aprendiz ativo, observador, formulador 
de perguntas, expressando livremente percepções e opiniões, principalmente por que é exposto à resolução de problemas reais, dando sentidos significativos à realidade vivenciada, o que propicia o desenvolvimento de habilidades de observação, análise, avaliação, compreensão e extrapolação, tornando o intercâmbio e a cooperação algo importante para sua aprendizagem que torna-se grupal, sem o foco no professor.

A concepção pedagógica da problematização deve levar em consideração a base comum de definições de problemas e significados, onde o indivíduo internaliza os conteúdos e significados a partir de sua própria experiência e histórias de apropriações, ou seja, sua subjetividade. ${ }^{16}$ Segundo estudiosos da área, deve-se valorizar a "busca de temas e questões centrais relatadas pelos indivíduos, entendidos mais como aqueles que geram motivação, emoção e envolvimento do que por sua frequência no relato" ${ }^{17}$.

Esta metodologia encontra fundamentos na filosofia de práxis, na pedagogia libertadora e problematizadora de autores como Paulo Freire e na pedagogia crítico-social dos conteúdos, que considera que a práxisimplica em ação e reflexão dos homens sobre o mundo para transformá-lo. ${ }^{14}$

Portanto, aprender é um ato de conhecimento da realidade concreta, da situação real vivida pelo educando, onde os educandos vão construindo sua compreensão em processo, em transformação. ${ }^{18}$

\section{Como foram"Os Encontros"}

Os encontros foram momentos de diálogo e de trocas de conhecimentos e experiências entre trabalhadores, gestores e docentes. No período de janeiro/2014 a julho/2015 foram promovidas sete reuniõeslocorregionais, com um turno (3 horas) de duração cada e periodicidade de 2 a 3 meses de intervalo. A data e horário foram previamente agendadas e pactuadas de forma a não prejudicar a rotina dos serviços.

Organizou-se ambiente mais tranquilo, fora das UBS, em uma sala de treinamentos da gestão municipal. Todos os médicos do PMM foram convidados a participar, assim comoa coordenação municipal da Saúde da Família, os supervisores e tutor da área. Conforme a temática, também os apoiadores municipais e especialistas da rede municipal foram convidados a discutir as situações problema em foco, os protocolos e linhas de cuidado utilizados, assim como os fluxos e questões relacionadas aos pontos de atenção da Rede de Atenção à Saúde da cidade.

A situação problema foi disparadora da discussão, quando, proposta pelos médicos e/ou ofertada pelos supervisores, possibilitava ampla análise e síntese coletiva sobre os temas. Com formação de pequenos grupos entre os participantes, a exposição deconhecimentos prévios sobre a questão trazida era o ponto de partida, para posteriormenteserem pactuadas hipóteses como fluxo de compreensão, avaliação como parâmetro de possibilidades e finalmente, um plano de cuidado como estratégia de solução. Em alguns temas, uma exposição dialogada de cerca de 30-40 minutos 


\section{$247 / /$}

foi realizada pelos supervisores ou especialista local, para sistematizar o conhecimento produzido pelo grupo, alinhando-o às práticas dos serviços do município. Considerando os atributos da APS, fomentava-se a ampliação do escopo de práticas, com cardápio de ofertas de serviços e o trabalho em equipe multiprofissional. A destacar a integração da abordagem clínica (em seus aspectos da promoção da saúde, prevenção e atenção), com a da saúde coletiva, de gestão (as particularidades dos territórios, o funcionamento da Rede, a vigilância em saúde).

Cabe destacar que, ao final dos encontros, reservávamos um tempo livre (30-40 mim) para discussão de questões administrativas e operacionais do PMM (férias, pagamentos, auxílio moradia, horários, dificuldades com a gestão local/equipe), aspectos que mobilizavam os médicos, mas que, se não organizados, tinham o potencial de ocupar em demasia o andamento das discussões sobre as temáticas propostas. Desta maneira um planejamento prévio da supervisão e tutoria foi essencial, tanto para construir a metodologia, as situações problemas, os objetivos de aprendizagem, quanto para elegermos as referências bibliográficas básicas. Assim reuniões presenciais e à distância, por meio de web conferência, foram norteadoras.

Realizou-se diagnóstico situacional dos territórios, levantamento de problemas de saúde do território, e identificação das lacunas do conhecimento (necessidades educacionais) por parte dos profissionais(2 encontros). Utilizou-se uma matriz de problemas e prioridades, com a seleção de temas para os encontros subsequentes, a saber:

- Saúde Mental (2 encontros): o primeiro, com a apresentação de casos clínicos trazidos pelos médicos (uso abusivo de benzodiazepínico; uso drogas ilícitas - crack- em adolescente); apoio matricial e rede de saúde mental; e no segundo, a discussão de casos complexos/transtorno mental possíveis de acompanhamento pela ESF, além dasistematização teórica de protocolos de diagnóstico e tratamento em saúde mental.

- Saúde da Mulher (1 encontro) com foco na saúde reprodutiva, a partir da discussão de casos, abordando planejamento familiar e pré-natal de baixo risco, também com a presença dos articuladores regionais visando a construção do cuidado em rede;

- Saúde Materno-infantil (1 encontro): a partir de um evento sentinela: morte de uma criança menor de um ano acompanhada por uma das equipes. O caso foi apresentado, discutindo-se a vulnerabilidade da família, os fatores de risco identificados no acompanhamento do pré-natal e no cuidado do bebê, além da apresentação da rede de atenção, também com a participação de articuladores regionais.

\section{Reflexões acerca das estratégias educacionais desenvolvidas:}

$\mathrm{Na}$ dimensão político-operacional, a construção das estratégias educacionais baseou-se na integração da gestão e supervisão no foco da educação permanente no campo da aplicabilidade em 
territórios com o modelo organizacional de atenção na saúde da família, considerando a construção de espaços de troca-sensibilização e pactuações. ${ }^{19}$

Com o processo formativo coletivo, buscou-se a implementação de práticas de atenção à saúde, requerendo novos pressupostos e métodos compartilhados para o enfrentamento ou a resolução de problemas. Partiu-se da compreensão do processo saúde-adoecimento com foco na integralidade e nas evidências clínicas e de gestão, trazendo-se ferramentas da gestão da clínica para potencializar o trabalho dos médicos vinculados a fomentos de provimento em áreas de vulnerabilidade social. ${ }^{20}$

Tal processo exigiu a problematização do cotidiano do trabalho, por meio das situações-problemas, que trouxerama perspectiva das visões dos diversos atores, possibilitando uma construção em rede. Entende-se que esta permite circular elementos materiais e imateriais entre cada uma de suas entidades, nem sempre com regras bem definidas, valorizando espaços de trocas, construção de acordos e pactuações de novas práticas.

$\mathrm{Na}$ definição sobre os temas das situações-problema, buscou-se trabalhar em sintonia com os problemas eleitos pelos médicos, considerando um tratamento crítico e reflexivo dos temas, com a flexibilidade dos pontos de estudos, seja através de encontros mais sistematizados como os encontros loco-regionais, seja nas visitas em campo, onde também abordávamos estes temas.

Assim, para trabalhar e problematizar a realidade da APS, a partir do conhecimento da realidade local, foi importante que os supervisores desenvolvessem um olhar atento às práticas dos médicos do PMM; olhar este potencializado pelas visitas realizadas, mas também pela escuta das prioridades eleitas pelo coletivo.

O envolvimento dos parceiros, gestores e atores-chavelocais culminou com a valorização da construção de uma rede, transformando os espaços coletivos das locorregionais em espaços de integração da própria Rede de Atenção àSaúde, constituídos como espaços de comunicação representativos de um movimento a favor da consolidação da APS. De forma ainda distante em sua plena operacionalização, se pensado na realidade brasileira, espera-se que a APS ordene os fluxos e contra fluxos de pessoas, produtos e informações, ao longo de todos os pontos de atenção à saúde e dos sistemas de apoio; e foi isto o que tentamos construir juntos.

A nosso favor tínhamos, certamente, o território, onde a população adscritatorna-se um dos elementos essenciais das redes de atenção, que, colocada sob responsabilidade sanitária, hierarquiza o processo de trabalho a partir de uma organicidade das famílias. ${ }^{21}$ Para avançar na construção da rede, é imprescindível a comunicação interpessoal, potencializada pela educação permanente dos profissionais de saúde e novamente, foi este o movimento que produzimos. No nosso caso, a participação da gestão e dos apoiadores municipais foi fundamental neste processo, já que se procurou,antes de tudo, estar em sintonia com as propostas e necessidades locais/regionais, aproveitando o espaço para o aprofundamento da práxis na saúde na construção de possíveis 
caminhos.

\section{CONSIDERAÇÕES FINAIS}

Concluímos que o trabalho de supervisão, ao se desenvolver em consonância com as diretrizes da Política Nacional de Educação Permanente em Saúde, considera as necessidades de formação dos profissionais, relacionaa complexidade da situação demográfica com atransição epidemiológica, além de possibilitar priorização da organizaçãodos sistemas de saúde locais.

Produzindo tensões e diálogos, o processo educativo provocoumudanças nas práticas dos profissionais e de suas equipes, contribuindo para qualificar o modelo de atenção à saúde do município. Dessa maneira, nosso trabalho na supervisão e tutoria foi se consolidando na medida em que construíamos espaços coletivos de troca, permitindo suavizar os previsíveis desencontros entre trabalhadores e gestão. Produzimos cuidado!

A educação permanente trabalha com pessoas adultas, o que requer um processo de ensino centrado no aprendiz, na independência e na autogestão da aprendizagem, já que, ao se aprender o que se precisa para aplicar na vida, constroem-se competências amplas, voltadas para o mundo do trabalho. Assim, nos parece que valorizamos as experiências acumuladas,onde o objetivo do aprendizado e sua aplicabilidade atenderamàs necessidades educacionais e laborais. ${ }^{22}$

Observamos que ao utilizarmos metodologias ativas de ensino aprendizagem, promovemos uma certa horizontalidade na relação professor (supervisor - facilitador)com o aprendiz (médico), o que valorizou a aprendizagem centrada em problemas e em experiências, como uma aprendizagem significativa, o que nos adequou com a proposta do fazer da Educação Permanente.

Outra grande potencialidade percebida foi o fato de que, através da metodologia de ensino aprendizagem adotada, conseguimos integrar médicos do PMM, apoiadores médicos e de outras categorias profissionais do município, tutor e gestores, em um movimento que enfrentouos nós críticos da rede e do modelo de atenção como um desafio de todos, gerando um sentido comum para o trabalho em saúde. Pensamos alternativas factíveis, sem secundarizar os eventuais embates, atravessados que são por escolhas políticas e gerenciais.

Como limitação, pode-se dizer que muitos dos problemas identificados estavam fora da governabilidade daquele coletivo, mas conhecê-los, identificá-los foi uma etapa essencial para o processo de solução de situações antes nem mesmo discutidas. E ainda, foi preciso tempo para experimentação e vivênciado método por parte dos trabalhadores, gestores, supervisores e tutor, todos aprendizes.

Consideramos importante avançar na integração do processo educativo, com aquele desenvolvido pela gestão municipal, incorporando outros médicos da rede básica e equipe multiprofissional, 
assim como a articulação com os conteúdos do curso de especialização que os médicos do PMM realizam.

Persiste o desafio de romper amarras de práticas clínicas estruturadas e exercitar a integração dos diversos profissionais envolvidos no cuidado, lidando com a divisão técnica e social do trabalho em equipe, um trabalho mais democrático e participativo apoiado em vários olhares. Desafios para um futuro próximo!

\section{REFERÊNCIAS BIBLIOGRÁFICAS}

1. Brasil. Ministério da Saúde. Secretaria de Atenção à Saúde. Departamento de Atenção Básica. Política Nacional de Atenção Básica / Ministério da Saúde. Secretaria de Atenção à Saúde. Departamento de Atenção Básica. - Brasília: Ministério da Saúde, 2012. 110 p.: il. - (Série E. Legislação em Saúde).

2.Brasil.Lei n. ${ }^{\circ}$ 12.871, de 22 outubro de 2013. Institui o Programa Mais Médicos, altera as Leis no 8.745 , de 9 de dezembro de 1993, e no 6.932, de 7 de julho de 1981, e dá outras providências. Diário Oficial da União, Brasília, DF, 23 out. 2013.

3. Brasil. Ministério da Saúde. Secretaria de Gestão do Trabalho e da Educação na Saúde. Programa Mais Médicos - dois anos: mais saúde para os brasileiros / Ministério da Saúde, Secretaria de Gestão do Trabalho e da Educação na Saúde - Brasília: Ministério da Saúde, 2015.

4.Brasil. UNASUS. Programa Mais Médicos. Relatório de supervisão. Disponível em: https:// sistemas.unasus.gov.br Acesso em: 28/9/2015.

5. Brasil. Ministério da Saúde. Secretaria de Gestão do Trabalho e da Educação na Saúde. Departamento de Gestão da Educação em Saúde. Política Nacional de Educação Permanente em Saúde. Brasília: Ministério da Saúde, 2006. 64 p. - (Série B. Textos Básicos de Saúde) (Série Pactos pela Saúde 2006; v. 9)

6. Brasil. Ministério da Saúde. Secretaria de Gestão do Trabalho e da Educação na Saúde. Universidade Aberta do Sistema Único de Saúde-UNASUS. Competências dos profissionais de nível superior na Estratégia de Saúde da Família. Brasília, DF, 2011.

7. StarfieldB.Atenção Primária: equilíbrio entre necessidades de saúde, serviços e tecnologia, Brasília: UNESCO, 2002.

8. Ceccim RB. O desenvolvimento de competências no trabalho em saúde: educação, áreas do conhecimento, e profissões no caso da saúde. Tempus: Actas de Saúde Coletiva,Abr.2012. 
9.Brasil.Ministério da Saúde. Portal Brasil. Mais Medicos atende 100\% das vagas no Rio de Janeiro. Disponívelem: http://www.brasil.gov.br/saude/2015/05/mais-medicos-atende-100-dasvagas-no-rio-de-janeiro._Acessoem 1/7/2015.

10. IBGE. Instituo Brasileiro de Geografia e Estatística. Estimativas populacionais para os municípios brasileiros. 2014.Disponível em http://www.ibge.gov.br/home/estatistica/populacao/ estimativa2014/

11.Bordenave JD, Pereira AM. Estratégias de Ensino-Aprendizagem. Petrópolis: Vozes, 23 ed.; 1977.

12. Dewey J. Como pensamos: como se relaciona o pensamento reflexivo com o processo educativo: uma reexposição. Rio de Janeiro: CompanhiaEditora Nacional. 1959.

13. Saviani DA. Questão Pedagógica na Formação de Professores. Florianopolis: Endipe, 1996.

14. Freire P. Pedagogia da Autonomia: saberesnecessários à práticaeducativa. São Paulo: Paz e Terra, 2009.

15. AusubelDP. A aprendizagem significativa: a teoria de David Ausubel. São Paulo: Moraes, 1982.

16. BerberNAN. A problematização e a aprendizagem baseada em problemas: diferentes termos ou diferentes caminhos? Interface-Comum, Saúde e Educação. Fevereiro, 1998. P.139-149 .

17. Pinto TR, Cyrino EG. Os profissionais de saúde no ensino na Atenção Primária à Saúde: tensões e potencialidade na práticas pedagógico-assistenciais. In: Cyrino AP; Godoy D \& Cyrino EG. Saúde, Ensino e Comunidade reflexões sobre práticas de ensino na atenção primária à saúde. São Paulo. Editora Cultura Acadêmica. 2014.p.93-132

18. Villardi ML, Cyrino EG, Berbel NAN.A metodologia da problematização no contexto da formação em saúde. In:Cyrino AP; Godoy D \& Cyrino, EG. Saúde, Ensino e Comunidade reflexões sobre práticas de ensino na atenção primária à saúde. São Paulo: Editora Cultura Acadêmica.2014. p.147-189.

19. Miranda VA, Monteiro VO, Teixeira SCS. Gestão, Supervisão e Educação Permanente em Territórios de Aprendizagem em Saúde da Família. In: MarinsJJN \& Rego S. Educação Médica: gestão, cuidado, avaliação. São Paulo: Hucitec Editora, Rio de Janeiro: Associação Brasileira de Educação Médica, 2011.p 203-222

20. OPAS.A Atenção à Saúde Coordenada pela APS: construindo as redes de Atenção no SUS: 
$252 / /$

contribuição para o debate/Organização Pan-Americana à Saúde.Brasília,2011. 113p

21. OPAS. A Atenção à Saúde Coordenada pela APS: construindo as redes de Atenção no SUS: contribuição para o debate/Organização Pan-Americana à Saúde.Brasília,2011. P.17

22. Lopes JMC et al. Manual de Oficina para Capacitar preceptores em Medicina de Família e Comunidade. Florianópolis: Sociedade Brasileira de Medicina de Família e Comunidade, 2009.p $55-63$

Artigo apresentado em 19-12-15 Artigo publicado no sistema em 05-05-16 Artigo aprovado em 06-04-16 